\title{
A medical report writer software
}

\section{Dr. M.H.B. Ariyaratne}

Postgraduate Institute of Medicine, University of Colombo, Sri Lanka.

E-mail address: buddhika.ari@gmail.com

eHealth Sri Lanka 2010,1(suppl.1):S31

DOI: http://dx.doi.org/10.4038/sljbmi.v1i0.3590

Only the Abstract is available

\begin{abstract}
Writing reports is a daily routine in several fields of clinical practice like Radiology and Pathology. These days computers are frequently used in writing reports using software like Microsoft Word. In order to add some flexibility to computer aided report writing, a small software called medical report writer was developed. Patient details like name, age, sex, ward and BHT can be easily entered. These will be included in the generated reports. Every detail entered like that can be used later to trace the report or to be used in various statistical analyses. These will be included in the generated reports.

After the initial set-up of the programme, writing a normal report is just a matter of a few clicks. For example, just select the normal CT Brain, and the format we have selected initially will be appearing. If the report is a commonly found abnormal finding, that can also be generated, for example, blood picture with reactive lymphocytosis. When the report is abnormal, what we can do it to click the relevant features from a list. After the report is generated, any change can be done manually. Checking the spellings and grammar is also available. A treasure is also available to be used when necessary. The complete report can be saved inside the programme for easy retrieval. Finally a print out can be taken or can be exported to Microsoft Word for further modification. The main advantages over using Microsoft Word include hassle free report saving, easier report retrieval, easier report generation and minimisation of naming errors.
\end{abstract}

Keywords - medical report writer, generated reports, statistical analyses 